\title{
On existence of a piecewise smooth cycle in one asymmetric gene network model with piecewise linear equations
}

\author{
V. Golubyatnikov ${ }^{1,2 *}$, L. Minushkina ${ }^{2}$ \\ ${ }^{1}$ Sobolev Institute of Mathematics SB RAS, Novosibirsk, Russia \\ ${ }^{2}$ Novosibirsk State University, Novosibirsk, Russia \\ *e-mail: vladimir.golubyatnikov1@fulbrightmail.org
}

Key words: Negative feedbacks, piecewise linear dynamical systems, invariant domains, cycles, state transition diagram

Motivation and Aim: We construct a simple piecewise linear dynamical system which simulates one gene network regulated by negative feedbacks in order to find conditions of existence of periodic regimes (cycles) of its functioning and to describe location of these cycles in the phase portrait of the system.

Methods and Algorithms: Some approaches to modelling of similar gene networks and description of combinatorial structures of discretizations (State Transition Diagram) of the phase portraits of corresponding nonlinear dynamical systems are presented in [1-3]. Results: For positive parameters $m_{j}, A_{j}$ and $\alpha_{j}$, where $A_{j}>\alpha_{j}, j=1,2,3$, we consider 3Ddynamical system

$\frac{d x}{d t}=L_{1}(z)-m_{1} x ; \frac{d y}{d t}=L_{2}(z)-m_{2} y ; \frac{d y}{d t}=L_{2}(y)-m_{3} z$.

Here $L_{j}$ are non-negative step-functions which correspond in gene network to negative feedbacks: $L_{j}\left(\left[0, \alpha_{j}\right)\right)=A_{j}$, and $L_{j}\left(\left[\alpha_{j}, \infty\right)\right)=0$. We show that trajectories of the system (1) are piecewise smooth, and that the polyhedral domain $Q=\left[0, A_{1}\right] \times\left[0, A_{2}\right] \times\left[0, A_{3}\right]$ is positively invariant with respect to shifts along these trajectories. Let us decompose this domain $Q$ to 8 smaller parallelepipeds by hyperplanes $x=\alpha_{1} ; y=\alpha_{2} ; z=\alpha_{3}$.

Theorem. There exists a piecewise smooth cycle $C$ of the system (1) which passes through union $U_{6}$ of 6 of these parallelepipeds $B_{k}$. The angle points of this cycle are located on the common faces of the parallelepipeds $B_{k}$.

So, this union $U_{6}$ is an invariant domain of the dynamical system (1) as well, it does not contain two parallelepipeds containing the origin and the "opposite" point $\left(A_{1}, A_{2}, A_{3}\right)$. The theorem follows from the analysis of linearization of the system (1) in each of the parallelepipeds $B_{k}$ near their common point $\left(\alpha_{1}, \alpha_{2}, \alpha_{3}\right)$. The existence of the cycle $C$ is shown with the help of the Brouwer fixed point theorem.

Conclusion: In contrast with [2], where the particular case $m_{1}=m_{2}=m_{1}=1$ was studied, the shifts along trajectories of the system (1) are not described by projective transformations of the faces of adjacent blocks $B_{k}$ which contain $\boldsymbol{C}$. Thus, the uniqueness of this cycle does not follow from the geometric arguments used in $[2,3]$.

Acknowledgements: Supported by RFBR, No. 18-01-00057.

References:

1. Likhoshvai V.A., Golubyatnikov V.P. et al. (2008) Theory of gene networks. In: System computerized biology. Novosibirsk, SB RAS, 397-480.

2. Ayupova N.B., Golubyatnikov V.P. (2014) On the uniqueness of a cycle in an asymmetric three-dimensional model of molecular repressilator. Journal of Applied and Industrial Mathematics. 8(2):1-6.

3. Ayupova N.B., Golubyatnikov V.P. (2015) On two classes of nonlinear dynamical systems: the 4-dimensional case. Siberian Mathematical Journal. 56(2):231-236. 